\title{
Nonlinear Fano resonance and bistable wave transmission
}

\author{
Andrey E. Miroshnichenko, ${ }^{1}$ Sergei F. Mingaleev, ${ }^{2,3}$ Sergej Flach, ${ }^{4}$ and Yuri S. Kivshar ${ }^{1}$ \\ ${ }^{1}$ Nonlinear Physics Centre and Centre for Ultra-high bandwidth Devices for Optical Systems (CUDOS), Research School of Physical \\ Sciences and Engineering, Australian National University, Canberra ACT 0200, Australia \\ ${ }^{2}$ Department of Physics, University of Central Florida, Orlando, Florida 32816, USA \\ ${ }^{3}$ Bogolyubov Institute for Theoretical Physics, Kiev 03143, Ukraine \\ ${ }^{4}$ Max-Planck-Insitut für Physik komplexer Systeme, Nöthnitzerstrasse 38, Dresden 01187, Germany
}

(Received 13 December 2004; published 30 March 2005)

\begin{abstract}
We consider a discrete model that describes a linear chain of particles coupled to a single-site defect with instantaneous Kerr nonlinearity. We show that this model can be regarded as a nonlinear generalization of the familiar Fano-Anderson model and it can generate amplitude-dependent bistable resonant transmission or reflection. We identify these effects as the nonlinear Fano resonance and study its properties for continuous waves and pulses.
\end{abstract}

DOI: 10.1103/PhysRevE.71.036626

PACS number(s): 42.25.Bs, 42.65.Pc, 42.65.Hw, 41.85.Ja

\section{INTRODUCTION}

The Fano resonance is widely known across many different branches of physics; it manifests itself as a sharp asymmetric profile of transmission or absorption lines, and it is observed in numerous physical systems, including light absorption by atomic systems [1], Aharonov-Bohm interferometers $[2,3]$, and quantum dots [4-6], resonant light propagation through photonic-crystal waveguides [7-12], and phonon scattering by time-periodic scattering potentials [13-15]. From the viewpoint of the fundamental physics, the Fano resonance may appear in systems characterized by a certain discrete energy state that interacts with the continuum spectrum through an interference effect. Usually, the discrete state is created by a defect that allows one (or several) additional propagation paths in the wave scattering which interact constructively or destructively. In the transmission line, this interference effect leads to either perfect transmission or perfect reflection, producing a sharp asymmetric profile.

In a classical paper, Fano [1] derived the general formula which describes asymmetric line shape of the transmission or absorption lines:

$$
\mathcal{F}(\epsilon)=\frac{(\epsilon+f)^{2}}{\epsilon^{2}+1},
$$

where $\epsilon=\left(E-E_{R}\right) /(\Gamma / 2)$ is the dimensionless energy in units of the resonance width $\Gamma, f$ is the asymmetry parameter (Fano factor), and $E_{R}$ is the resonance energy. In the limit $f \rightarrow \infty$, this formula can also describe the so-called BreitWigner resonance profile [2]. This quite universal formula is used usually to fit a particular profile observed in experiments and, therefore, to provide proof that the observed phenomenon can be classified as the Fano resonance.

One of the simplest models that can describe the resonant coupling and interaction between a discrete state and continuum spectrum is the so-called Fano-Anderson model [16] (see a sketch in Fig. 1). It describes a linear "atomic" chain with the nearest-neighbor interaction forces and interacting with a defect state through the nearest neighbors. In application to tight-binding models in solids, these forces are linked to a hopping probability. This simple model allows one to describe the basic physics of the Fano resonance in a simple way. In particular, this model allows some analytical studies that may serve as a guideline for the analysis of more complicated physical models that predict the Fano resonance effect.

In this paper we consider an important generalization of the Fano-Anderson model under the assumption that a single-site defect is nonlinear. We show that this model allows one to describe a nonlinear Fano resonance, and it can generate extremely high contrast between the bistable states in its transmission with low input power. We study resonant nonlinear transmission and the properties of the nonlinear Fano resonance for continuous waves and pulses. We would like to mention that a typical physical situation when this model can be employed directly for describing resonant effects is light transmission in waveguides and waveguide junctions created in two-dimensional photonic crystals with embedded high- $Q$ resonators (defects or cavities) with nonlinear response. In the nonlinear regime, a defect supports one (or several) localized states that are characterized by a discrete eigenvalue. When the excited localized state interacts with the photonic-crystal waveguide, the system can display interference effects and Fano resonance.

The aim of this paper is twofold. First, we study both linear and nonlinear transmission in discrete Fano-Anderson models and obtain a number of explicit analytical results

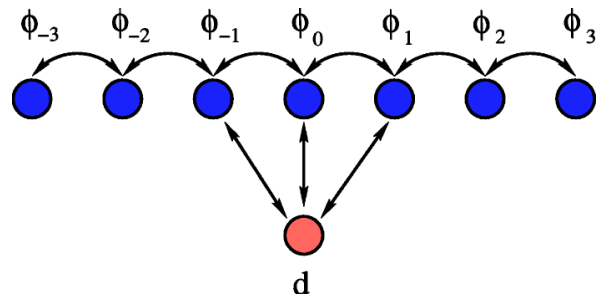

FIG. 1. (Color online) Schematic of the Fano-Anderson model. The array of blue circles corresponds to a linear chain, and the isolated red circle is a defect which can be either linear or nonlinear. Arrows indicate the coupling between different states. 
which allow one to get a deeper understanding of the physics of this phenomenon. Second, we analyze in detail the effect of nonlinearity on the Fano resonance and demonstrate that, first, it leads to a shift of the Fano resonance frequency and, second, it can lead to bistability in the wave transmission.

The paper is organized as follows. In Sec. II we describe our discrete nonlinear model which provides a generalization of the Fano-Anderson model. In Sec. III, we describe the main features of the Fano resonance for the case of a local coupling and then study the effect of nonlinearity of the defect on the resonant transmission. In particular, we define the conditions for the bistable transmission and show that the nonlinear Fano resonance can exist in a broad frequency range. Section IV is devoted to the study of a model with nonlocal coupling in both the linear and nonlinear regimes. In Sec. V we consider wave packet scattering. Section VI concludes the paper.

\section{MODEL}

One of the simplest models which describes the physics and main features of the Fano resonance is the so-called Fano-Anderson model [16]. In this paper, we use a modified version of this model described by the Hamiltonian (see Fig. 1)

$$
H=\sum_{n} C \phi_{n} \phi_{n-1}^{*}+E_{d}|d|^{2}+\lambda|d|^{4}+d^{*} \sum_{j} V_{j} \phi_{j}+\text { c.c. },
$$

where the asterisk denotes complex conjugation. This model describes the interaction of two subsystems. One subsystem consists of a straight linear chains with complex field amplitude $\phi_{n}$ at site $n$ which are coupled by nearest-neighbor coupling $C$; this is the system characterized by the frequency band of the continuum spectrum. The second subsystem consists of an additional discrete state $d$ with local energy value $E_{d}$. For the nonlinear model, we assume that the defect possesses a cubic nonlinear response, $\lambda$ being the nonlinear parameter. The interaction between these two subsystems is described by the coupling coefficients $V_{j}$.

From the lattice Hamiltonian, we derive a system of coupled nonlinear dynamic equations

$$
\begin{gathered}
i \dot{\phi}_{n}=C\left(\phi_{n-1}+\phi_{n+1}\right)+d \sum_{j} V_{j} \delta_{n j}, \\
i \dot{d}=E_{d} d+\lambda|d|^{2} d+\sum_{j} V_{j} \phi_{j},
\end{gathered}
$$

where the overdot stands for the derivative in time. For further analysis, we look for stationary solutions of this system in the form

$$
\phi_{n}(\tau)=A_{n} e^{-\mathrm{i} \omega \tau}, \quad d(\tau)=B e^{-\mathrm{i} \omega \tau},
$$

which allow us to describe elastic scattering processes by means of a system of nonlinear algebraic equations

$$
\begin{gathered}
\omega A_{n}=C\left(A_{n-1}+A_{n+1}\right)+B \sum_{j} V_{j} \delta_{n j}, \\
\omega B=E_{d} B+\lambda|B|^{2} B+\sum_{j} V_{j} A_{j} .
\end{gathered}
$$

The model (4) is the main subject of our analysis of stationary-wave scattering. In the following two sections, we study first the regime of a local coupling when only one coupling coefficient $V_{0}$ is nonzero and after that consider a nonlocal coupling with up to three nonzero coupling coefficients $V_{-1,0,1}$. In both these cases, we start our analysis from the linear regime and then analyze the Fano scattering in the nonlinear regime when $\lambda \neq 0$. A full time-dependent model (2) is discussed in Sec. V of the paper when we consider wave packet scattering.

\section{LOCAL COUPLING}

First, we analyze the case of a local coupling when $V_{j}$ $\equiv 0$ for $j \neq 0$ and $V_{0}$ is nonzero and study separately the linear $(\lambda=0)$ and nonlinear $(\lambda \neq 0)$ regimes.

\section{A. Linear scattering}

We consider the scattering of plane waves with dispersion $\omega_{q}=2 C \cos q$ propagating along the linear chain. Using the second equation of the system (4), we find a simple link between two defect-site characteristics,

$$
B=\frac{V_{0} A_{0}}{\omega-E_{d}},
$$

and obtain a single equation

$$
\omega A_{n}=C\left(A_{n-1}+A_{n+1}\right)+\frac{V_{0}^{2} A_{0}}{\omega-E_{d}} \delta_{n 0},
$$

with a one-site scattering potential. For the scattering problem, we consider the boundary conditions

$$
A_{n}= \begin{cases}I e^{\mathrm{i} q n}+r e^{-\mathrm{i} q n}, & n<0, \\ t e^{\mathrm{i} q n}, & n>0,\end{cases}
$$

where $I, r$, and $t$ have the meaning of the incoming, reflected, and transmitted wave amplitudes far from the defect site. Without loss of generality, we will assume that the incoming amplitude $I$ is real. According to Eq. (6), the strength of the scattering potential depends on the incoming frequency $\omega$, and the system should demonstrate resonant scattering. If the frequency of the defect is placed in the propagation frequency band of the chain-i.e., $\left|E_{d}\right| \leqslant 2 C$ - the scattering potential in Eq. (6) becomes infinitely large at $\omega \equiv \omega_{F}=E_{d}$, and this will lead to total reflection of the incoming wave.

Using the well-known transfer-matrix approach, we obtain the analytical result for the transmission coefficient defined as $T=|t|^{2} / I^{2}$ :

$$
T=\frac{\alpha_{q}^{2}}{\alpha_{q}^{2}+1},
$$

where $\alpha_{q}=c_{q}\left(\omega_{q}-E_{d}\right) / V_{0}^{2}$ and $c_{q}=2 C \sin q$. This result corresponds to a simple physics: For the resonant frequency $\omega_{F}$, there exist two scattering channels, with and without the defect state excited, and destructive interference between the waves passing these channels leads to a complete suppression of the wave transmission (see Fig. 2). As a matter of 


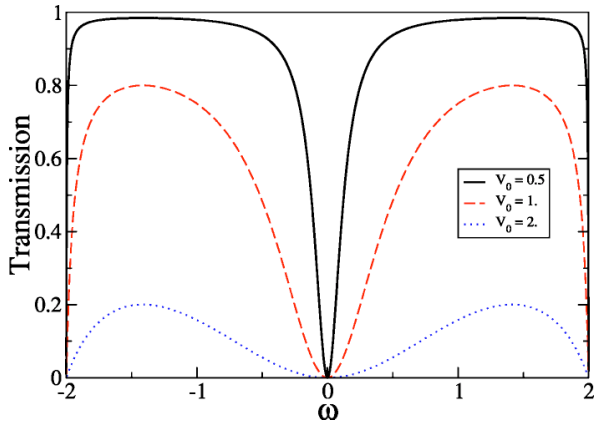

FIG. 2. (Color online) Transmission coefficient (8) calculated for the linear Fano-Anderson model (2) in the case $E_{d}=0, C=1$, and $\lambda=0$, for several values of the coupling coefficient $V_{0}$.

fact, the result (8) corresponds to the Fano formula (1), where $\alpha_{q}$ plays a role of the dimensionless energy, $E_{d}$ is the resonant energy eigenvalue, and the resonance width is defined as

$$
\Gamma=\frac{V_{0}^{2}}{C \sin q_{F}},
$$

where $q_{F}$ is a wave number at the resonance, $\omega_{F}=\omega\left(q_{F}\right)$. According to this result, the width is proportional to the coupling strength $V$, and it depends on the position of the Fano resonance with respect to the boundary of the spectrum [17]. In this case, the asymmetry parameter $f$ vanishes, and the transmission profile is symmetric.

Transmission vanishes when $\alpha_{q}=0$; this happens at the edges of the continuous spectrum band, $q=0$ and $q=\pi$, due to the vanishing of group velocities, and at the resonant frequency $\omega_{F}$ which, in the case of a local coupling, coincides with the discrete eigenvalue of the defect mode, $\omega_{F}=E_{d}$. If the defect is coupled to two or more sites, the resonant frequency is renormalized, as discussed below. We notice that when the resonant frequency is in the middle of the propagation band, the transmission profile is symmetric (see Fig. 2 ), and in the case of a local coupling the Fano resonance is observed as the resonance reflection.

Using that $\left|A_{0}\right|^{2}=T I^{2}$ and the relation (5), we can calculate analytically the amplitude of the defect state, which riches the maximum

$$
\left|B_{\max }\right|^{2}=I^{2} / 2 \Gamma
$$

at the Fano resonance frequency $\omega_{F}$.

When the coupling coefficient between the defect and chain vanishes, $V_{0}=0$, linear modes of the system (4) are described by the continuous spectrum $\omega_{q}=2 C \cos q$, while the defect with frequency $\omega=E_{d}$ is uncoupled. For a finite coupling $V_{0} \neq 0$, the defect generates two local modes (or bound states) with the frequencies bifurcating from the upper and lower edges of the continuous spectrum, $\left|\omega_{L}\right| \geqslant 2 C$, as shown in Fig. 3. The appearance of these local modes does not show any characteristic feature in transmission close to the band edges. For the case $E_{d}=0$, these states are located symmetrically, because the defect frequency is at the middle of the spectrum $\omega_{q}$.

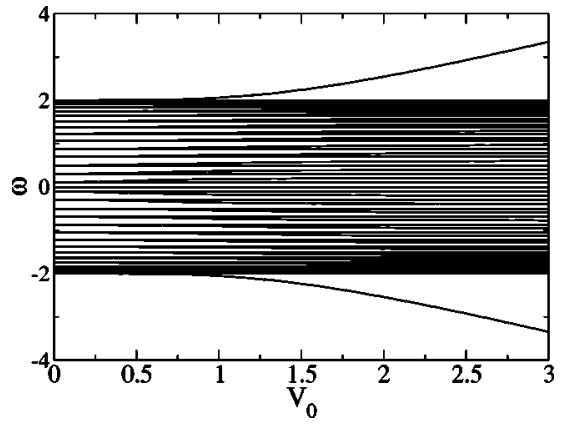

FIG. 3. Frequency spectrum of the system (4) vs the coupling parameter $V_{0}$ for the case $C=1, E_{d}=0$, and $\lambda=0$.

In general, the frequency of the localized state $\omega_{L}$ of the one-site potential is given by the expression $\omega_{L}^{2}=4 C^{2}+\xi^{2}$, where $\xi$ is the strength of the potential, and in our case,

$$
\omega_{L}^{2}=4 C^{2}+\frac{V_{0}^{4}}{\left(\omega_{L}-E_{d}\right)^{2}} .
$$

Formally, this is the fourth-order equation in $\omega_{L}$, but for $E_{d}$ inside the spectrum $\omega_{q}$, there exist only two real solutions which correspond to two bound states.

\section{B. Nonlinear scattering and bistability}

Now we assume that the isolated defect is nonlinear-i.e., $\lambda \neq 0$. Using Eqs. (4) and the continuity condition at the defect site, $I+r=t$, we obtain the general expression for the transmission coefficient,

$$
T=\frac{x^{2}}{x^{2}+1},
$$

where $x$ is a real solution of the cubic equation

$$
\left(x^{2}+1\right)\left(x-\alpha_{q}\right)-\gamma_{q}=0,
$$

with the parameter $\gamma_{q}=\lambda c_{q}^{3} I^{2} / V_{0}^{4}$.

The transmission coefficient defined by Eq. (12) corresponds again to the general Fano formula (1). During our derivation, we put $x=\tan \theta$, where $\theta$ is a phase of the reflection amplitude $r=|r| e^{\theta}$. Due to the local range of the scattering potential (6), it can be shown [19] that the phase of the reflection amplitude is equal to $\theta=\delta(q)+\pi / 2$, where $\delta(q)$ is a scattering phase shift. Therefore, we can consider Eq. (13) as a nonlinear equation for the scattering phase shift, with $x=-\cot \delta(q)$. Note here that Eq. (13) may support complex solutions for scattering phase shifts, which will correspond to inelastic scattering [19]. Such a situation is beyond the scope of this paper. Expression (12) is valid only for real solutions of Eq. (13), which correspond to elastic scattering process.

Transmission (12) vanishes at $x=0$ when $\alpha_{q}=-\gamma_{q}$ :

$$
V_{0}^{2}\left(E_{d}-\omega_{q}\right)+\lambda I^{2}\left(4 C^{2}-\omega_{q}^{2}\right)=0
$$

or $c_{q}=0$.

When $\gamma_{q}=0$, there exists only one real solution of Eq. (13), $x=\alpha_{q}$; it leads to the result (8) obtained above. In the nonlinear regime $\left(\gamma_{q} \neq 0\right)$, there exist up to three real solu- 


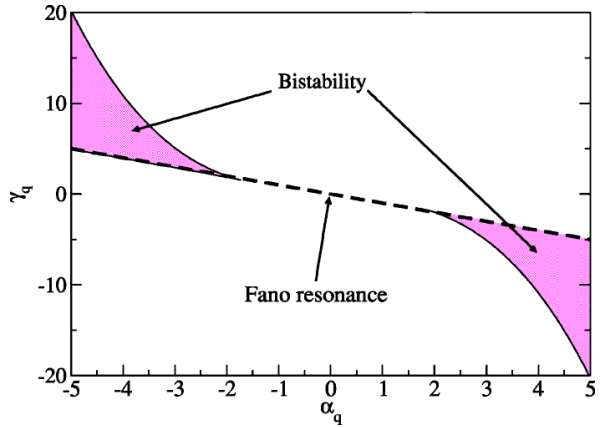

FIG. 4. (Color online) Parameter plane $\left(\alpha_{q}, \gamma_{q}\right)$ defined by different solutions of Eq. (13). Solid areas correspond to bistability; a straight dashed line at the boundary of the bistability region corresponds to the Fano resonance.

tions, the output may become a multivalued function of the input, and this may lead to bistability of the wave transmission [20].

To define the regions where such a bistability transmission can occur, we present the left-hand side of Eq. (13) in the form $F(x)=f(x)-\gamma_{q}$, so that the bistability regions are determined by local extrema of the function $f(x)$. When $\left|\alpha_{q}\right|<\sqrt{3}$ there are no local extrema, and for any value of $\gamma_{q}$ (and also $\lambda$ or $I$ ) there exists only one real solution of Eq. (13) and therefore the transmission is always single valued. For $\left|\alpha_{q}\right|>\sqrt{3}$, a pair of minimum and maximum points of the function $f(x)$ appear at $x_{\text {min, } \max }=\left(\alpha_{q} \pm \sqrt{\alpha_{q}^{2}-3}\right) / 3$, respectively, and the bistability region is determined by the condition

$$
f\left(x_{\min }\right)<\gamma_{q}<f\left(x_{\max }\right),
$$

filled on the parameter plane $\left(\alpha_{q}, \gamma_{q}\right)$ in Fig. 4.

In the nonlinear regime, the transmission coefficient depends on two parameters: the wave frequency $\omega_{q}$ and input intensity $I$. First, we study the dependence of the transmission coefficient on the wave frequency. From the second equation of Eqs. (4), we can see that nonlinearity shifts the frequency of the localized mode and, therefore, shifts the position of the Fano resonance. By denoting the left-hand side of the Eq. (14) as $g\left(\omega_{q}\right)$, it follows that $g\left(\omega_{q}\right)$ is a quadratic function of $\omega_{q}$. If we put $E_{d}=0$, then $g(-2 C) g(2 C)$ $<0$ and, therefore, there is a unique solution of $g\left(\omega_{q}\right)$ inside the interval $[-2 C, 2 C]$. In the limit of large intensity-i.e., for $I \rightarrow \infty$ - the solutions of the equation $g\left(\omega_{q}\right)=0$ approach the values $\pm 2 C$. Thus, for any value of the input intensity $I$ there always exists a single Fano resonance [see Fig. 5(a)].

To define the position of the Fano resonance analytically, we solve Eq. (14) explicitly with respect to the frequency $\omega_{q}$ :

$$
\omega_{F}=-\frac{V_{0}^{2} \pm \sqrt{V_{0}^{4}+4 \lambda I^{2}\left(V_{0}^{2} E_{d}+4 \lambda I^{2} C^{2}\right)}}{2 \lambda I^{2}} .
$$

A proper sign in Eq. (16) should be chosen depending on the particular values of the parameters.

Thus, by increasing the input intensity, we shift the position of Fano resonance such that for $I>I_{\mathrm{cr}}$, the transmission coefficient becomes multivalued and a bistability region ap-
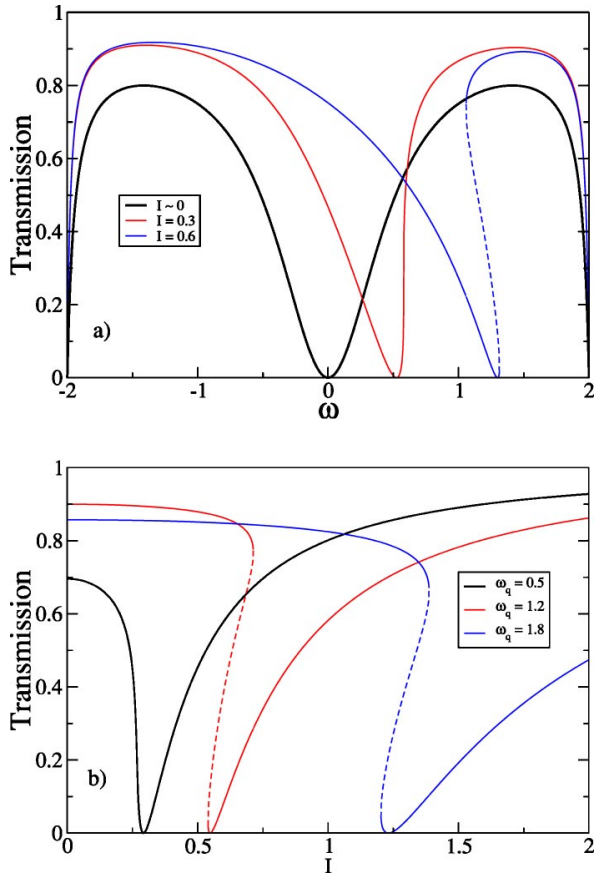

FIG. 5. (Color online) Dependence of the transmission coefficient on (a) the wave frequency $\omega_{q}$ for a fixed intensity $I$ and (b) input intensity $I$ for a fixed frequency $\omega$, for $C=\lambda=1, V_{0}=0.8$, and $E_{d}=0$. Bistability regions $\left(\left|\alpha_{q}\right|>\sqrt{3}\right)$ are denoted by dashed lines.

pears. Because both $\alpha_{q}$ and $\gamma_{q}$ depend on the frequency $\omega_{q}$, a change of the frequency corresponds to a move in the parameter space $\left(\alpha_{q}, \gamma_{q}\right)$. For $E_{d}=0, \alpha_{q}$ changes in the interval $\left[-\alpha_{q}^{\max }, \alpha_{q}^{\max }\right]$, where

$$
\alpha_{q}^{\max }=2 C^{2} / V_{0}^{2}
$$

and bistability is expected for $\alpha_{q}^{\max }>\sqrt{3}$ [see Fig. 5(a)].

To study the dependence of the transmission coefficient on the input intensity $I$, we solve Eq. (14) and obtain

$$
I^{2}=\frac{\left(\omega_{q}-E_{d}\right) V_{0}^{2}}{\lambda\left(4 C^{2}-\omega_{q}^{2}\right)} .
$$

Equation (18) indicates that, depending on the sign of nonlinearity $\lambda$, the Fano resonance appears for any frequency from the interval $\left[-2 C, E_{d}\right]$ or $\left[E_{d}, 2 C\right]$, and the frequency should satisfy the condition

$$
\left(E_{d}-\omega_{q}\right) \lambda<0 .
$$

On the plane of parameters $\left(\alpha_{q}, \gamma_{q}\right)$, only $\gamma_{q}$ depends on the input intensity $I$, so that varying $I$ we move along the line $\alpha_{q}=$ const and $\gamma_{q}<0$ or $\gamma_{q}>0$ (depending on the sign of the nonlinearity parameter $\lambda$ ). In order to achieve bistability, we should satisfy the conditions $\left|\alpha_{q}\right|>\sqrt{3}$ and $\alpha_{q} \gamma_{q}<0$. The latter condition can be reduced to the inequality $\left(E_{d}-\omega_{q}\right) \lambda$ $<0$, which defines the existence of the Fano resonance (19). In other words, bistability appears only simultaneously with the presence of Fano resonance; see Figs. 5(b) and 4. 


\section{NONLOCAL COUPLING}

As a matter of fact, the local coupling discussed above provides a relatively simple approach for the derivation of analytical formulas and understanding the major physics of the Fano resonance. However, very often the physical problems where the Fano resonance is observed are more complicated. In particular, one of the major properties of realistic physical models such as photonic-crystal waveguides coupled to localized defect modes is long-range interaction and nonlocal coupling [18]. Below, we study the generalized discrete (linear and nonlinear) Fano-Anderson model where the defect is coupled to three nearest neighbors of the chain-i.e., in Eq. (2), $V_{j} \neq 0$ for $j=-1,0,1$.

\section{A. Linear scattering}

In order to find the transmission coefficient for a nonlocal model, we modify the boundary conditions, Eq. (7):

$$
A_{n}= \begin{cases}I e^{\mathrm{i} q n}+r e^{-\mathrm{i} q n}, & n<-1, \\ t e^{\mathrm{i} q n}, & n>1 .\end{cases}
$$

In addition, we should solve a set of coupled equations for the sites $n=-1,0,1$ with the boundary conditions (20) with respect to the reflection coefficient $R=|r|^{2} / I^{2}$ :

$$
R=\frac{\left[\operatorname{Re}\left(b_{q}\right)\right]^{2}}{\left[\operatorname{Re}\left(b_{q}\right)\right]^{2}+\left[c_{q}\left(\omega_{q}-E_{d}\right)+\operatorname{Im}\left(b_{q}\right)\right]^{2}},
$$

where $b_{q}=V_{-1}^{2}+V_{0}^{2}+V_{1}^{2}+2 e^{2 \mathrm{i} q} V_{-1} V_{1}+2 e^{\mathrm{i} q} V_{0}\left(V_{-1}+V_{1}\right)$. The transmission coefficient $T$ can be found from the identity $T$ $+R=1$.

In the nonlocal case, the transmission coefficient displays more complex behavior as compared with the case of a onesite coupling (see Sec. III above). In particular, in this case the Fano resonance is asymmetric. Perfect reflection occurs when the second term in the denominator of Eq. (21) vanishes. The corresponding condition can be rewritten in the form

$$
c_{q}\left[C\left(\omega_{q}-E_{d}\right)+V_{0}\left(V_{-1}+V_{1}\right)+2 \cos q V_{1} V_{-1}\right]=0,
$$

and it is satisfied at the spectrum band edges $q=0$ and $q$ $=\pi$, due to zero group velocities and also when

$$
\left.\omega\right|_{T=0}=\frac{C^{2} E_{d}-C V_{0}\left(V_{-1}+V_{1}\right)}{C^{2}+V_{1} V_{-1}} .
$$

Equation (22) shows that, in the case of nonlocal coupling to a defect state, the position of the perfect reflection gets shifted. If the coupling is strong enough, the resonance can move outside the spectrum band.

In addition, there exists the frequency when a perfect transmission occurs. This happens when $\operatorname{Re}\left(b_{q}\right)=0$ or

$$
\left.\frac{\omega}{C}\right|_{T=1}=\frac{-V_{0}\left(V_{1}+V_{-1}\right) \pm \sqrt{\left(V_{0}^{2}-4 V_{1} V_{-1}\right)\left(V_{1}-V_{-1}\right)^{2}}}{2 V_{1} V_{-1}},
$$

and the corresponding frequency $\left.\omega\right|_{T=1}$ does not depend on the discrete state energy $E_{d}$. This means when the position of

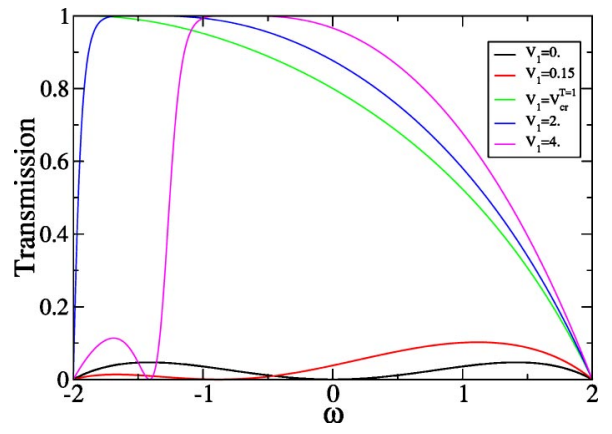

FIG. 6. (Color online) Transmission coefficient $T$ vs frequency $\omega_{q}$ for the three-site interaction, for different values of $V_{1}=V_{-1}$. Other parameters are $C=1, V_{0}=3$, and $E_{d}=0$. According to Eq. (25), perfect transmission starts to occur inside the spectrum for $V_{1}>1.5$. There is an interval for coupling coefficient $V_{1}$, when there is no perfect reflection inside the spectrum. For larger values of $V_{1}$, both perfect transmission and perfect reflection move to the middle of the spectrum $\omega_{q}=0$.

perfect reflection $\left.\omega\right|_{T=0}$ is shifted, the point of the perfect transmission remains unchanged. This property allows us to vary the width of the asymmetric Fano resonance by changing the energy $E_{d}$.

Formally, there exist two solutions for perfect transmission. But in real systems, such as waveguides in photonic crystals, some symmetries of the coupling coefficients hold-i.e., $V_{1}=V_{-1}$ or $V_{-1}=V_{0}$ and $V_{1}=0$-in the cases of three and two nonzero coupling terms, correspondingly. For the first case $V_{1}=V_{-1}$, we obtain

$$
\left.\omega\right|_{T=1}=-C\left(V_{0} / V_{1}\right)
$$

and therefore only one solution for perfect transmission is possible. For small values of $V_{1}$, there is no perfect transmission, and it takes place at the boundary of the spectrum $\left.\omega\right|_{T=1}=-2 C$, when

$$
V_{1}=V_{c r}^{T=1} \equiv V_{0} / 2
$$

and it exists for any larger value of $V_{1}$. When $V_{1} \rightarrow \infty$, the frequency of the perfect transmission moves to the middle of the spectrum, $\left.\omega\right|_{T=1} \rightarrow 0$ (see Fig. 6).

In the nonlocal model, the linear spectrum varies substantially as a function of the coupling coefficient $V_{1}$; see Fig. 7. For small values of $V_{1}$, two localized states exist outside the linear spectrum band $\omega_{q}$ (cf. Fig. 3). The frequency of the upper localized state goes away from the spectrum for all $V_{1}$. The lower localized state approaches the spectrum band but then deviates again for larger $V_{1}$.

Levinson's theorem [21] allows us to connect some properties of the linear spectrum (Fig. 7) with the transmission coefficient (Fig. 6). According to this theorem the scattering phase shift at one of the band edges can be represented as

$$
\delta(q=0)=\pi\left(N_{b}+l / 2\right),
$$

where $N_{b}$ is a number of bound states of the system and $l$ is a number of quasibound states at the band edge. Using the relation between transmission coefficient and scattering phase shift $[19,21]$, it can be shown that $T(q=0)=0$ for even 


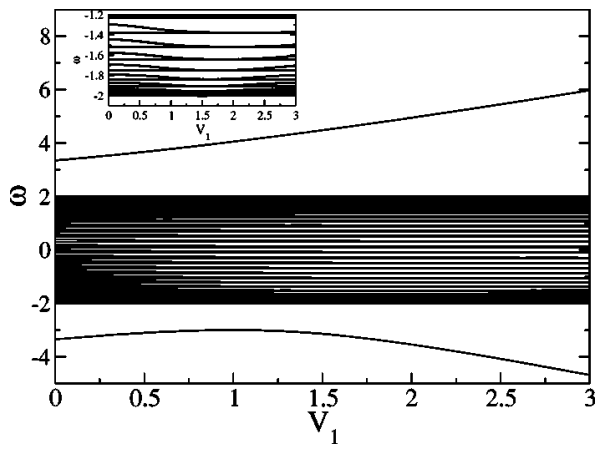

FIG. 7. Frequency spectrum for the nonlocal model vs coupling coefficient $V_{1}$ for the same values of parameters as in Fig. 6. The inset shows a zoomed-in area with the double degeneracy of the energy levels near the critical value $V_{\mathrm{cr}}^{T=1}$.

and $T(q=0)=1$ for odd values of $l$. Therefore, we can expect that some quasibound states should appear at the band edge, when condition (25) is satisfied. The inset in Fig. 7 shows that exactly for this value of the coupling parameter $V_{1}$, the spectrum band edge becomes doubly degenerate. In this situation $l=1$.

For the second case $V_{0}=V_{-1}$ and $V_{1}=0$, we obtain the result

$$
\left.\omega\right|_{T=1}=-2 C,
$$

so that the position of perfect transmission does not depend on the coupling coefficients and it should take place at the boundary of the spectrum band. According to Levinson's theorem, this may correspond only when some quasibound state exists exactly at the band edge, $l=1$ in Eq. (26).

Figure 8 predicts the perfect transmission at $\omega_{q}=-2 C$ for all values of $V_{0}$, except the value

$$
V_{0}=V_{\mathrm{cr}}^{T=0} \equiv \sqrt{C\left(E_{d}+2\right)},
$$

when the perfect reflection (22) splits off the spectrum band (see Fig. 8). In this case, for any nonzero value of $V_{0}$ there exists an upper localized state and the quasibound state at the

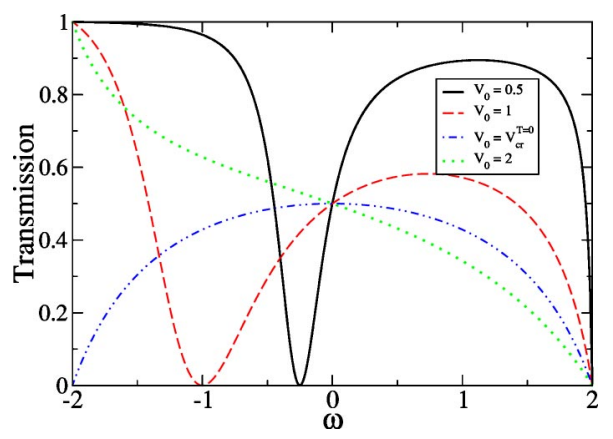

FIG. 8. (Color online) Transmission coefficient $T$ vs frequency $\omega_{q}$ for the nonlocal model with two nonzero couplings for different values of coupling $V_{0}=V_{-1}$. Other parameters are $C=1$ and $E_{d}=0$. Perfect transmission occurs at $\omega_{1}=-2$. When $V_{0}=V_{\mathrm{cr}}^{T=0}=\sqrt{2}$, the perfect reflection state splits off the spectrum band and transmission at $\omega=-2$ vanishes. Note here that for the given parameters $T(\omega=0)$ $=1 / 2$ for any value of the coupling $V_{0}$.

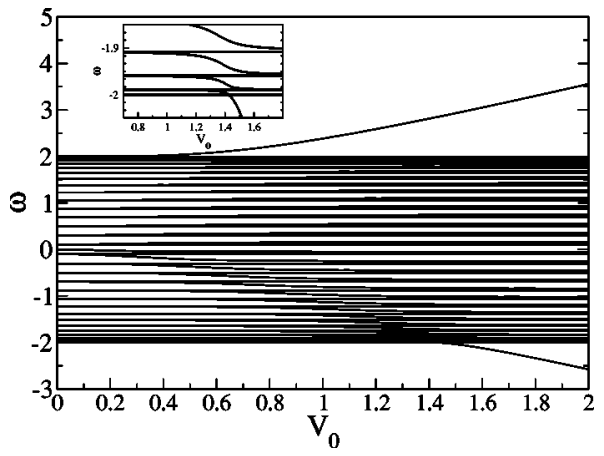

FIG. 9. Frequency spectrum for the nonlocal model with two nonzero coupling terms $V_{0}=V_{-1}$ vs the coupling coefficient $V$ for the same parameters as in Fig. 8. The inset shows a localized state at $\omega=-2$, which does not interact with other modes. The second bound state appears at $V_{0}=V_{\mathrm{cr}}^{T=0}$ when the perfect reflection splits off the spectrum band.

band edge $\omega=-2 C$ (see Fig. 9). In addition to this, at $V_{0}$ $=V_{\mathrm{cr}}^{T=0}$ another quasibound state appears at the band edge of the spectrum. In such a situation there are two quasibound states $(l=2)$ and according to Levinson's theorem (26) transmission coefficient vanishes at the bottom band edge. For larger values of coupling $V_{0}$ a second bound state exists in the system.

\section{B. Nonlinear scattering}

In the nonlinear regime, the reflection coefficient can be found in the analytical form

$$
R=\frac{\left[\operatorname{Re}\left(a_{q}^{2}\right)+\operatorname{Im}\left(a_{q}^{2}\right) x\right]^{2}}{\left[\operatorname{Re}\left(b_{q}\right)\right]^{2}\left(1+x^{2}\right)},
$$

where $x$ is a solution of the cubic equation

$$
\lambda I^{2} c_{q}^{3} y_{x}^{3}-\left(x^{2}+1\right)\left[\operatorname{Re}\left(b_{q}\right)\right]^{2}\left[\left|a_{q}\right|^{2} \operatorname{Re}\left(b_{q}\right) z_{x}+e_{q} y_{x}\right]=0,
$$

where $y_{x}=\operatorname{Re}\left(a_{q}^{2}\right)+\operatorname{Im}\left(a_{q}^{2}\right) x, \quad z_{x}=\operatorname{Re}\left(a_{q}^{2}\right) x-\operatorname{Im}\left(a_{q}^{2}\right), \quad a_{q}=V_{-1}$ $+e^{i q}\left(V_{0}+e^{i q} V_{1}\right), e_{q}=\left|a_{q}\right|^{2}\left[\left(\omega_{q}-E_{d}\right) c_{q}+\operatorname{Im}\left(b_{q}\right)\right]$, and the symbols $\mathrm{Re}$ and Im stand for the real and imaginary parts, respectively.

We would like to mention again that the general result for the reflection coefficient (29) is similar to the Fano formula (1), where $x$ can be understood as the effective dimensionless energy $\epsilon$ and the ratio $\operatorname{Re}\left(a_{q}^{2}\right) / \operatorname{Im}\left(a_{q}^{2}\right)$ is related to the asymmetry parameter $f$. This formula (29) includes all other cases discussed above. The condition for the perfect transmission $\operatorname{Re}(b)=0$ coincides with the result (23), so that the frequency of the perfect transmission does not depend on the input intensity $I$, nonlinearity parameter $\lambda$, and the energy of the discrete state $E_{d}$. The position of the perfect reflection does depend on all these parameters, and this gives us with a possibility to vary the width of the asymmetric resonance in the nonlocal nonlinear regime.

Finally, in Fig. 10 we plot the dependence of the transmission coefficient versus input intensity $I$ for different values of the frequency. These results are similar to those ob- 


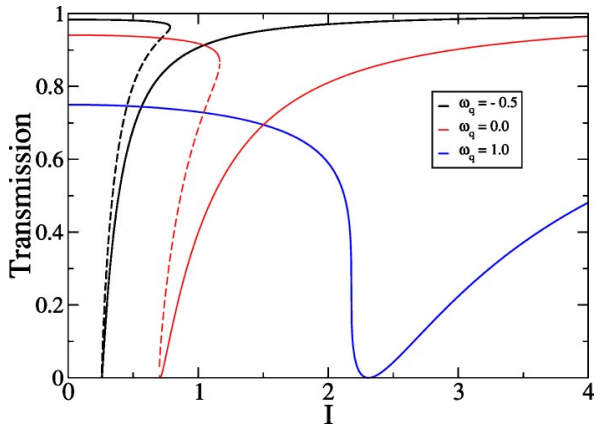

FIG. 10. (Color online) Transmission coefficient vs intensity $I$ for the nonlocal nonlinear model for $V_{-1}=V_{0}=V_{1}=1, \lambda=1, C=1$, and $E_{d}=0$. Perfect reflection occurs at the edge of the bistability domain (see Fig. 4). Dashed lines correspond to bistable regions.

tained for the model with a local coupling, presented in Fig. $5(b)$.

\section{WAVE PACKET SCATTERING}

The previous sections are devoted to the problem of timeindependent plane-wave scattering. In order to verify how these results can be used for describing realistic wave scattering, we should analyze the time-dependent scattering of wave packets. In this section, we study this problem numerically in the framework of the time-dependent model (2).

We consider the propagation of a Gaussian wave packet through a discrete chain with the defect described by the system of equations (2) in the regime of a local coupling, when $V_{0}=V$ and $V_{n \neq 0}=0$. As the input wave, we take a Gaussian wave packet of the form

$$
\phi_{n}(0)=I_{w} \exp \left[-\frac{\left(n-n_{0}\right)^{2}}{\sigma^{2}}\right] \exp \left[-i q_{w}\left(n-n_{0}\right)\right],
$$

where $q_{w}$ is the carrier wave number of the wave packet, $I_{w}$ is its maximum amplitude, $\sigma$ is the spatial width, and $n_{0}$ is the initial position. The wave number $q_{w}$ determines the velocity of the wave packet.

For calculating the transmission and reflection coefficients, we use the conservation of the norm and define the coefficients as follows:

$$
T=\frac{\sum_{n>0}\left|\phi_{n}\left(\tau^{*}\right)\right|^{2}}{\sum_{n}\left|\phi_{n}(0)\right|^{2}}, \quad R=\frac{\sum_{n<0}\left|\phi_{n}\left(\tau^{*}\right)\right|^{2}}{\sum_{n}\left|\phi_{n}(0)\right|^{2}},
$$

where $\tau^{*}$ corresponds to some time after interaction of the wave packet with a defect.

In our simulations, we take $E_{d}=0$ and therefore $q_{F}=\pi / 2$. First, we compare numerical results for wave packet scattering with plane-wave analysis in the linear regime (see Fig. 11 ). When the spectral width of the wave packet $2 \pi / \sigma$ is smaller than the width of the resonance (9), we have observed good agreement.

In the nonlinear regime, our numerical results for wave packet scattering are summarized in Fig. 12 for the dependence of the transmission and reflection coefficients on the

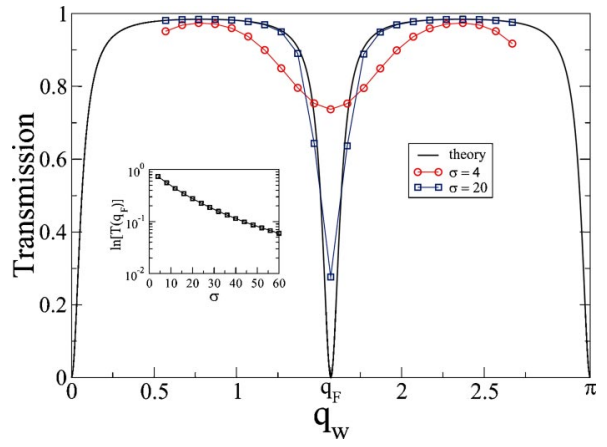

FIG. 11. (Color online) Transmission coefficient of the wave packet in the linear regime $\lambda=0$ for different values of the width $\sigma$. Other parameters are $C=1, V=0.5$, and $E_{d}=0$. For given parameters the spectral width of the wave packet becomes smaller than the width of the resonance $\Gamma=1 / 4$ for $\sigma>20$. It leads to good agreement with the analytical result. (Inset) Transmission at the position of the Fano resonance $q_{F}$ decays exponentially with the spatial width $\sigma$.

input wave packet amplitude $I_{w}$ defined in Eq. (31) for different values of the carrier wave number. For the case $q_{w}$ $<q_{F}$, Fig. 12 indicates the existence of the Fano resonance characterized by the resonant deep in the transmission coefficient and simultaneous resonant peak in the reflection coefficient. When $q_{w}>q_{F}$, no Fano resonance exists. These results provide a good qualitative agreement with the planewave analysis presented above.

In addition to this feature associated with the Fano resonance, there are observed some regions in Fig. 12 when both transmission and reflection coefficients decrease-i.e., when their sum does not equal unity. Usually, the discrete localized mode becomes excited during the interaction of a wave packet with the defect, and then it relaxes to the ground state. For these particular values of the wave packet amplitude $I_{w}$, both the defect-site and zero-site particles of the chain get highly excited with long-lived oscillations after the interaction (see Fig. 13). Such a behavior is very similar to the scattering by a $\delta$ potential with an excited state [19] and it corresponds to the inelastic scattering process. In our situation the localized state is excited due to the nonlinear interaction.

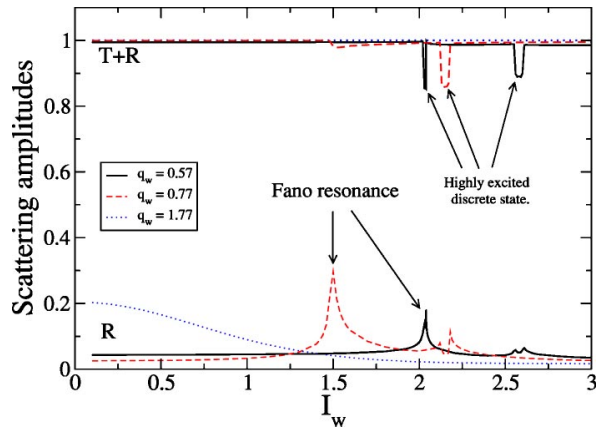

FIG. 12. (Color online) Reflection coefficient and the sum of the transmission and reflections coefficients vs the input amplitude $I_{w}$ of the wave packet defined in Eq. (31) for $C=1, V=0.5, E_{d}=0, \lambda$ $=1, \sigma=4$, and $n_{0}=-10$. 


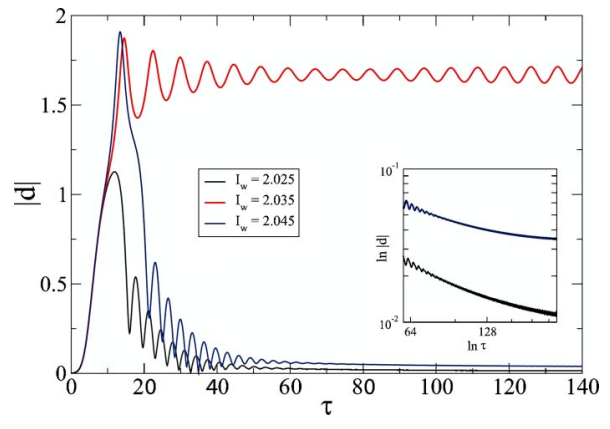

FIG. 13. (Color online) Evolution of the defect site during and after wave packet scattering, for $q_{w}=0.57$. Other parameters are the same as in Fig. 12. Different final states of the defect are observed for a slight variation of the amplitude of the incoming wave packet, being a signature of the system bistability. The inset shows a logplot of this dependence and indicates that the defect state relaxes to the ground state algebraically.

For the wave number $q_{w}=0.57$ such a situation even happens near the Fano resonance; i.e., by varying the amplitude of the wave packet we can observe different outcomes for the dynamics of the discrete state (see Fig. 13).

\section{CONCLUSIONS}

We have studied the properties of wave transmission in the presence of a localized nonlinear defect described by the generalized discrete Fano-Anderson model. This model provides a simple generalization of the well-known problem of Fano resonances to the nonlinear case, and it can be employed to describe the so-called nonlinear Fano resonance effect. We have studied, both analytically and numerically, the main features of the resonant transmission for continuous waves and pulses, and we have shown that the Fano resonance is observed as a specific feature in the transmission coefficient as a function of frequency and/or intensity. In particular, the presence of nonlinearity makes the resonance more robust and it can broaden substantially the parameter region where the resonant transmission is observed, by adjusting either the wave frequency or the intensity to satisfy the resonant conditions. Moreover, we have found the conditions when the Fano resonance is associated with the bistable transmission.

We would like to emphasize that many of the effects described in this paper can find applications in a variety of different nonlinear physical systems, including the transmission of straight and curved waveguides coupled to localized defect modes in photonic crystals $[8,9,18]$, plasmariton resonance in the nonlinear optics (see Fig. 12 in Ref. [22]). Moreover, a number of interesting effects already observed in some of the nonlinear systems can be identified as the manifestation of the nonlinear Fano resonances. In particular, we believe that the resent observation of excitonic optical bistability in $\mathrm{Cu}_{2} \mathrm{O}$ [23] can be explained in terms of the nonlinear Fano resonance which could shed additional light on the physics of these experimental observations.

\section{ACKNOWLEDGMENTS}

This work has been supported by the Australian Research Council. We thank B. Malomed, S. Fan, M. Soljacic, and A. Gorbach for useful discussions. A part of this work has been conducted under the research projects of the ARC Centre of Excellence CUDOS.
[1] U. Fano, Phys. Rev. 124, 1866 (1961).

[2] J. U. Nöckel and A. D. Stone, Phys. Rev. B 50, 17415 (1994).

[3] K. Kobayashi, H. Aikawa, S. Katsumoto, and Y. Iye, Phys. Rev. Lett. 88, 256806 (2002).

[4] J. Göres, D. Goldhaber-Gordon, S. Heemeyer, M. A. Kastner, H. Shtrikman, D. Mahalu, and U. Meirav, Phys. Rev. B 62, 2188 (2000).

[5] B. R. Bulka and P. Stefanski, Phys. Rev. Lett. 86, 5128 (2001).

[6] M. E. Torio, K. Hallberg, S. Flach, A. E. Miroshnichenko, and M. Titov, Eur. Phys. J. B 37, 399 (2004); A. A. Aligia and L. A. Salguero, Phys. Rev. B 70, 075307 (2004).

[7] S. Fan and J. D. Joannopoulos, Phys. Rev. B 65, 235112 (2002).

[8] S. H. Fan, Appl. Phys. Lett. 80, 908 (2002).

[9] M. F. Yanik, S. H. Fan, and M. Soljacic, Appl. Phys. Lett. 83, 2739 (2003).

[10] A. R. Cowan and J. F. Young, Phys. Rev. E 68, 046606 (2003).

[11] S. H. Fan, W. Suh, and J. D. Joannopoulos, J. Opt. Soc. Am. B 20, 569 (2003).

[12] V. Lousse and J. P. Vigneron, Phys. Rev. B 69, 155106 (2004).
[13] E. Tekman and P. F. Bagwell, Phys. Rev. B 48, 2553 (1993).

[14] S. W. Kim and S. Kim, Phys. Rev. B 63, 212301 (2001).

[15] S. Flach, A. E. Miroshnichenko, V. Fleurov, and M. V. Fistul, Phys. Rev. Lett. 90, 084101 (2003).

[16] G. D. Mahan, Many-Particle Physics (New York, Plenum Press, 1993).

[17] A. E. Miroshnichenko, S. Flach, and B. A. Malomed, Chaos 13, 874 (2003).

[18] S. F. Mingaleev and Yu. S. Kivshar, J. Opt. Soc. Am. B 19, 2241 (2002).

[19] H. J. Lipkin, Quantum Mechanics: New Approaches to Selected Topics (North-Holland, Amsterdam, 1973).

[20] H. M. Gibbs, Optical Bistability: Controlling Light with Light, (Academic Press, New York, 1985).

[21] C. J. Joachain, Quantum Collision Theory (North-Holland, Amsterdam, 1975).

[22] T. Andersen, O. Keller, W. Hübner, and Börje Johansson, Phys. Rev. A 70, 043806 (2004).

[23] G. Dasbach, G. Baldassarri Höger von Högersthal, D. Frölich, H. Stolz, and M. Bayer, Phys. Rev. B 70, 121202(R) (2004). 